\title{
Intrauterine death: an approach to the analysis of genetic heterogeneity
}

\author{
E BOTTINI, L COROMALDI, E CARAPELLA, R PASCONE, M NICOTRA, \\ I COGHI, N LUCARINI, AND F GLORIA-BOTTINI
}

From the Interdisciplinary Centre for Applied Mathematics, Lincei National Academy, Rome; the Department of Genetics, University of Camerino, Camerino; and the Departments of Child Health and Obstetrics and Gynaecology, University of Rome, Rome, Italy.

SUMMARY A study of survival time of zygotes in utero and the relationship with parental phenotype $\stackrel{\sim}{\circ}$ of a series of genetic polymorphisms was carried out in 41 couples with habitual abortion. Variability $\stackrel{\omega}{\vec{U}}$

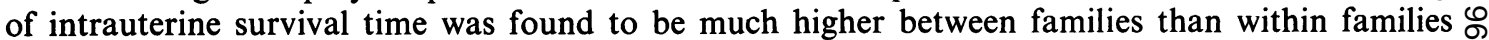
suggesting that several genetic entities contribute to the condition clinically defined as habitual 9 abortion. Significant differences of survival time were found in relation to the length of the paternal $\vec{c}$ $\mathrm{Y}$ chromosome and to the maternal phenotypes of $\mathrm{PGM}_{1}$ and Ss. These observations are in line $\stackrel{\complement}{\subseteq}$ with previous data suggesting intrauterine selection in these polymorphisms. Further studies of the $\mathbb{D}$ timing of intrauterine death in relation to 'normal' genetic polymorphisms may help to clarify the $\vec{\varphi}$ aetiology of spontaneous fetal loss.

Recent studies indicate that the overall death rate of zygotes ranges between 25 and $75 \% .^{12}$ Besides environmental agents, genetic factors are considered to be of paramount importance in this phenomenon. ${ }^{2-5}$ As in other phases of life, it is likely that both monogenic and polygenic heredity play an important part in intrauterine survival. Genetic factors may also play a role in predisposing to chromosomal aberration frequently observed in clinically recognisable spontaneous abortion. ${ }^{3}$

It is well known that genotypes expressing their deleterious effects after birth show a characteristic chronological pattern of manifestations. The timing of clinical manifestations and death is usually different between genotypes and very similar within families. By analogy, the study of survival time of zygotes in women with repeated abortion not resulting from anatomical or other known environmental causes, and the analysis of relationships with genetic factors statistically associated with fetal death, may improve the understanding of the aetiology of intrauterine death.

\section{Material and methods}

Forty-one couples who had had at least two consecutive abortions were examined. A total of 140 abortions was recorded.

Received for publication 29 September 1982. Accepted for publication 30 October 1982.
In general, after two consecutive abortions, the following examinations are carried out: hystero- $\bar{o}$

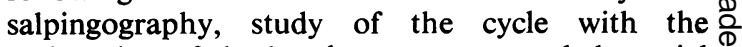
registration of the basal temperature and the serial $\triangle$ determination of serum progesterone levels during $\overrightarrow{\overrightarrow{0}}$ the luteinic phase, glucose tolerance test, serological 3 tests for lues, and toxoplasma tests. At least three examinations of the seminal fluid are carried out as? well.

Tests for thyroid function, endometrium biopsies, and tests for cytomegalovirus, mycoplasms, and $\frac{5}{3}$ listeria are carried out only in selected cases. Psychological investigations and help (frequent $\delta$ visits and availability at all times) are offered to all couples with habitual abortions. In the couples included in the present analysis all these clinical $>$ examinations were consistently negative.

Gestational duration of the first aborted fetus was $N$ always estimated from the date of the last menstrual or period as recorded from the patient's memory. For subsequent miscarriages, ovulation date was $\underset{\omega}{\tilde{O}}$ generally determined by basal temperature charts.

In all couples a karyotype analysis was carried out 0 with $C$ or $G$ banding ${ }^{6}$ or both and the phenotype $\overparen{D}$ of the following systems was determined by standard $\stackrel{\oplus}{+}$ techniques: ABO, Rh, MNSs, Secretor (Se), 뭉 haptoglobins $(\mathrm{Hp})$, phosphoglucomutase $\left(\mathrm{PGM}_{1}\right)$, adenosine deaminase (ADA), and adenylate kinase ? $\left(\mathrm{AK}_{1}\right) .^{7}$ The HLA phenotype (nine antigens for the $\stackrel{\mathbb{\Omega}}{\stackrel{1}{2}}$ $A$ locus and 15 antigens for the B locus) was 196 
TABLE Gestational duration in habitual abortion. Relation with parental phenotype of some genetic polymorphisms.

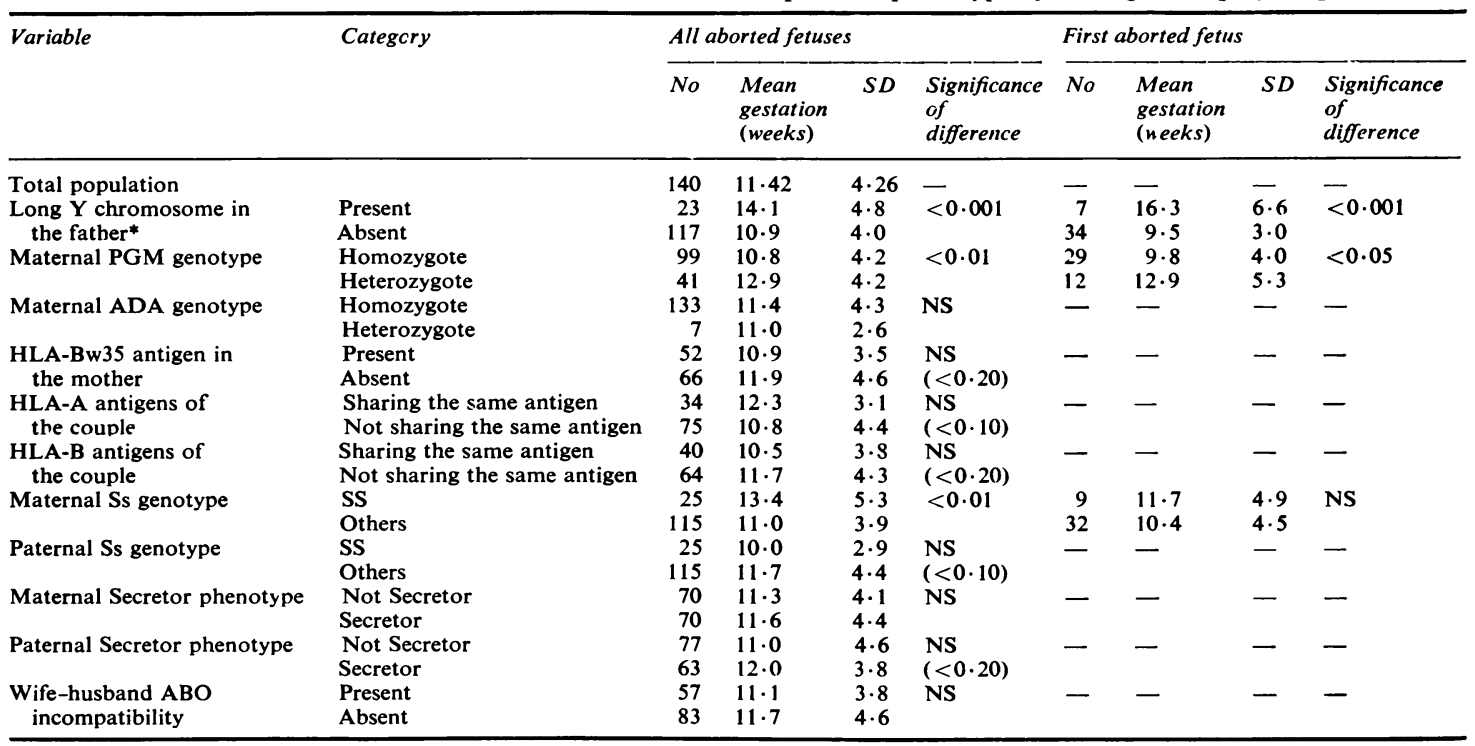

*Criterion adopted for defining a $\mathrm{Y}$ chomosome as long was a ratio $\mathrm{Y} / \mathrm{F} \geqslant \mathbf{1 \cdot 0 .}$

determined in 35 couples. Preliminary data concerning the phenotype distribution of some of these systems have been published elsewhere. ${ }^{8-11}$

Variables included in the present analysis are reported in the table. Selection of variables and their subdivision into categories was based on statistical association with spontaneous abortion observed in our sample or reported by other investigators. ${ }^{8-14}$ Statistical analyses were carried out using the SPSS programme with a $370 / 158$ IBM computer. ${ }^{15}$

\section{Results}

The distribution of the mean gestationa duration calculated for each couple with habitual abortion is depicted in the figure. Couples were quite uniformly distributed over the range observed in our sample. Variability of intrauterine survival time was much higher between families than within families. Variance analysis showed significant heterogeneity among couples.

The relation between gestational duration and some parental genetic variables is analysed in the table. Significant differences in intrauterine survival time were observed in relation to the length of the paternal $\mathrm{Y}$ chromosome and maternal $\mathrm{PGM}_{\mathbf{1}}$ and Ss phenotypes. The same pattern of associations was observed after examination of only the first aborted fetus of the couples.

Variance analysis showed no interaction between maternal PGM $_{1}$ phenotype, maternal Ss phenotype,

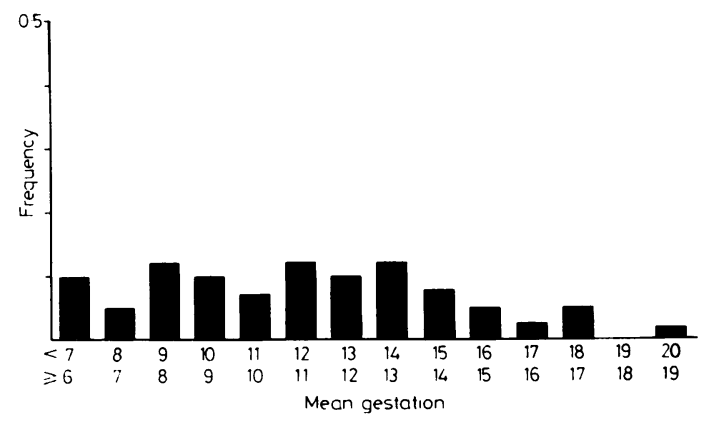

FIGURE Distribution of mean duration of gestation (weeks) in couples with habitual abortion.

Variance analysis. Total number of abortions $=140$

$\begin{array}{lll} & \text { Mean square } & d f \\ \text { Between families } & 31 \cdot 3363 & 40 \\ \text { Within families } & 12 \cdot 8355 & 99\end{array}$

$\mathrm{F}=2 \cdot 4414, \mathrm{p}<0.01$.

99

and length of $\mathrm{Y}$ chromosome in the father in their effects on gestational duration of aborted fetuses.

\section{Discussion}

The distribution of the mean gestational durations and the heterogeneity between families are compatible with the conjecture that several genetic entities contribute to the condition clinically defined 
as habitual abortion. 'Normal' genetic polymorphisms seem at present to be the most interesting candidates for the analysis of multifactorial variables influencing intrauterine development and survival. ${ }^{16}$

Association with spontaneous abortion has been observed for ABO, ${ }^{12} \mathrm{HLA},{ }^{8}{ }^{13} \mathrm{Pl},{ }^{17} \mathrm{MNSs}^{9}{ }^{9} \mathrm{PGM}_{1},{ }^{10}$ Secretor (Bottini et al, unpublished observations), ADA,11 and chromosomal variants. ${ }^{14}$ The observation of a relationship between gestational duration and a given polymorphism reinforces the statistical inference based on the increased proportion of a particular phenotype in spontaneous abortion. If a given genotype predisposes to fetal loss, a temporal pattern of recurrence could be associated with that genotype. The relevant phenomenon is the existence of a temporal distribution of fetal losses different from that observed among other genotypes. A decrease of intrauterine survival in comparison to other lethal conditions would not necessarily be expected.

The present analysis supports the view that $\mathrm{PGM}_{1}$, Ss, and structural variation of the $Y$ chromosome influence intrauterine selection and survival.

Our approach has not shown significant results for ADA, HLA, ABO, and Secretor. Several hypotheses for this can be put forward. (1) Statistical association previously reported was fortuitous, (2) the size of the present sample is not sufficient to reveal slight differences of gestational duration, and (3) these factors exert a protective action during the whole span of gestation and therefore lack a typical temporal pattern of manifestations.

The selection of couples with repeated abortions may have definite advantages for our study. While the correct assignment of a single episode of abortion as spontaneous or induced is generally difficult, our cases are certainly all spontaneous and it is likely that our sampling method selects families genetically predisposed.

The exact ascertainment of gestational duration is difficult; this, however, should not affect the results of our analysis since the error is likely to be independent of the genetic marker investigated. The fact that the pattern of relationships between the gestational durations and the polymorphisms was the same when considering only the first aborted fetuses (for which the exact ascertainment of gestational duration was more difficult) supports this view.

Although many more families should be studied in order to draw definite conclusions, our observations suggest that the analysis of genetic polymorphisms in relation to the timing of fetal death may give insight into the aetiology of habitual abortion and into the identification of the forces which maintain genetic variability.

This work was supported by CNR.

\section{References}

1 Miller JF, Williamson E, Glue J, Gordon YB, 心 Grudzinskas JG, Sykes A. Fetal loss after implantation- $\vec{\circ}$ a prospective study. Lancet 1980 ;ii:554-6.

2 Abramson FD. Spontaneous fetal death in man. Soc Biol $1974 ; 20: 375-403$

3 Lauritsen JG. Genetic aspect of spontaneous abortion. Thesis, University of Aarhus, 1977.

4 Snow MHC. Maternal effects on development. Nature 1976;260:94.

5 Lauritsen JG. Aetiology of spontaneous abortion. A $\dot{\omega}$ cytogenetic and epidemiological study of 288 abortuses and their parents. Acta Obstet Gynaecol Scand 1976; कू suppl 52.

6 Priest JH. Medical cytogenetics and cell culture. 을 Philadelphia: Lea and Febiger, 1977.

7 Harris H, Hopkinson DA. Handbook of enzyme electrophoresis in human genetics. Amsterdam: North Holland, 1976.

8 Purpura M, Coghi I, Nicotra M, Carapella E, Bottini E. $\vec{\bullet}$ HLA Bw35 antigen and human reproduction. J Med Genet 1980;17:157.

9 Carapella E, Purpura M, Coghi I, Bottini E. Blood groups and histocompatibility antigens in habitual abortion. Haematologia (Budap) 1980;13:105-11.

10 Lucarini N, Discepoli L, Nicotra M, Pascone R, Balducci M, Falsi AM. Ricerca di selezione sul polimorfismo del 응 PGM $_{1}$ durante la vita intrauterina. Atti AGI 1979;24: 188-9.

11 Bottini E, Carapella E, Cataldi L, et al. Adenosine deaminase polymorphism. Associations at clinical level suggest a role in cell functions and immune reactions. J Med Genet $1981 ; 18: 331-4$.

12 Takano K, Miller JR. ABO incompatibility as cause of spontaneous abortion: evidence from abortuses. $J$ Med Genet 1972;9:144-50.

13 Komlos L, Zamir R, Joshua H, Halbrecht I. Common HLA antigens in couples with repeated abortions. Clin Immunol Immunopathol 1977;7:330-5.

14 Patil SR, Lubs HA. A possible association of long $Y$ chromosomes and fetal loss. Hum Genet 1977;35:233-5.

15 Nie NH, Hull CH, Jenkins JG, Steinbrenner K, Bent DH. ㄱ SPSS. Statistical package for the social sciences. New York: McGraw-Hill, 1975.

16 Bottini E, Orzalesi M, Carapella E, Gloria-Bottini F. Genetic polymorphism, intrauterine selection and $\mathrm{N}$ perinatal morbidity. Rev Perinatal Med 1981;4:189-219.

17 Beckman G, Beckman L, Magnusson SS. Placental O alkaline phosphatase phenotypes and pre-natal selection. N Hum Hered 1972;22:473-80.

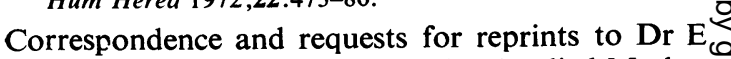
Bottini, Interdisciplinary Centre for Applied Mathe- $\frac{\bar{D}}{\mathbb{D}}$ matics, Accademia Nazionale dei Lincei, Rome, $\stackrel{\mathscr{S}}{\rightarrow}$ Italy. 